TRABAJO ORIGINAL

\title{
Asociación del peso al nacer con adiposidad en niños escolares de distintas regiones de argentina
}

\author{
Association of birth weight with adiposity in school \\ children from different regions of argentina
}

\begin{abstract}
Valeria Hirschler ${ }^{1}$, Edit Scaiola², Claudio González³, Zelmira Guntsche ${ }^{4}$ Claudia Molinari ${ }^{5}$, Cecilia Miorin ${ }^{6}$, Natacha Maldonado7 , Silvia Gorbán de Lapertosa ${ }^{8}$, Concepción García9 Mariana Hidalgo ${ }^{10}$, Gustavo Maccallini ${ }^{11}$, Agustina Lizazú ${ }^{12}$, Valeria Calzia ${ }^{7}$, Romina Di Firma ${ }^{7}$, Ornella Lione $^{13}$, Gianluca Sansoni ${ }^{12}$, Patricia Bocco ${ }^{14}$, María Carolina Albornoz ${ }^{15}$, Alejandra Verónica Burgarello ${ }^{15}$, Lorena Beatriz Benitez ${ }^{15}$, Silvina Radio ${ }^{16}$, Carolina Beatriz Yulán ${ }^{17}$, Silvana Beatriz Cáceres ${ }^{18}$, Mariana Andrea De La Rosa ${ }^{19}$, Sabrina Martín ${ }^{20}$, Julieta Pomilio ${ }^{20}$, Belén Raina ${ }^{20}$, Pilar Márquez ${ }^{21}$, Yamil Pareja ${ }^{21}$, Antonella Debiasi ${ }^{22}$, Martín Muzaber23, Gina Pipistrelli24, Carla Pons ${ }^{24}$, Diana Lang ${ }^{24}$. En nombre del proyecto DIOSES.
\end{abstract}

\section{RESUMEN}

Introducción: diversos estudios han sugerido que el bajo y alto peso al nacer (PN) se asocian a obesidad (OB) y sobrepeso (SP) durante la infancia y la edad adulta.

Objetivos: determinar la asociación entre PN y OB en escolares de 9 años de tres regiones de Argentina.

Materiales y métodos: las medidas antropométricas y la presión arterial (PA) se tomaron en 1.131 escolares (505 masculinos) de 8,8 $\pm 2,1$ años de edad promedio, en tres regiones de Argentina durante el año 2019. Se interrogó, además, acerca del peso al nacer y el estilo de vida.

Resultados: el 21,1\% (239) de los niños presentaba SP (IMC>85 <95 percentilo según CDC) y el 21,8\% (246) OB (IMC>95 percentilo). La prevalencia de PN bajo $(<2.500 \mathrm{~g})$ fue de fue de $6,2 \%(n=70)$ y de PN alto $(>4.000 \mathrm{~g})$ de $7,3 \%(n=82)$. El puntaje z-IMC de los niños de 9 años aumentaba significativamente con el aumento del PN: PN bajo (z-IMC=0,33), normal $(z-I M C=0,72)$ y alto $(z-I M C=1,12)$. En modelos de regresión logística múltiple se observó que el PN bajo se asoció inversamente a la OB (OR, 0.41 [IC del 95\%: 0,19-0,92]), mientras que el PN alto se asoció directamente con la OB ajustado por edad y sexo (OR, 2.48 [95\% IC 1,53-4,02]).

Conclusiones: nuestros datos indican que el alto PN, pero no el bajo PN, se asocia con OB en niños en edad escolar de 9 años, mientras que el bajo PN está inversamente asociado con OB.

Palabras clave: peso de nacimiento; obesidad; sobrepeso; escolares; estilo de vida.

Revista de la Sociedad Argentina de Diabetes 2020; Vol. 54 (125-131)

\section{ABSTRACT}

Introduction: several studies have suggested that low and high birth weight are associated with obesity (OB) and overweight (OW) during childhood and adulthood.

Objectives: to determine the association between birth weight and $O B$ in 9-year-old schoolchildren from three areas of Argentina.

Materials and methods: anthropometric measurements and blood pressure (BP) were taken in 1.131 schoolchildren $(505$ males) of an average age of $8.8 \pm 2.1$ years in three areas of Argentina during 2019. Mothers were asked about their children's birth weight and lifestyle.

Results: $21.1 \%$ (239) of the children had OW (BMI>>5 <95 percentile according to the CDC) and 21.8\% (246) OB (BMI>95 percentile). The prevalence of low birth weight $(<2.500 \mathrm{~g})$ was $6.2 \%(n=70)$ and of high birth weight $(>4.000 \mathrm{~g})$ was $7.3 \%$ $(n=82)$. The 9-year-old $z$-BMI score increased significantly with increasing birth weight: low birth weight (z-BMI=0.33), normal $(z-B M I=0.72)$ and high $(z-B M I=1.12)$. In multiple logistic regression models, it was found that low birth weight was inversely associated with OB (OR, 0.41 [95\% Cl: 0.19-0.92]), while high birth weight was directly associated with $O B$ adjusted for age and sex (OR, 2.48 [95\% Cl 1.53-4.02]).

Conclusions: our data indicate that high birth weight, but not low birth weight, is associated with OB in 9-year-old schoolchildren, while low birth weight is inversely associated with $O B$.

Key words: birth weight; obesity; overweight; schoolchildren; lifestyle.

Revista de la Sociedad Argentina de Diabetes 2020; Vol. 54 (125-131)
Médica Pediatra, especialista en Nutrición y Diabetes, Universidad de Buenos Aires, Coordinadora del Comité de Epidemiología, Sociedad Argentina de Diabetes, Ciudad Autónoma de Buenos Aires, Argentina

2 Médica Pediatra, consultorio privado, ex Médica Pediatra del Hospital Regional Ushuaia, ex Jefa del Departamento Materno Infantil, Tierra del Fuego, Argentina

3 Médico Farmacólogo, Departamento de Farmacología, Ins- tituto Universitario CEMIC, Ciudad Autónoma de Buenos Aires, Argentina

4 Médica Pediatra, especialista en Endocrinología, Servicio de Endocrinología, Hospital Notti, Mendoza, Argentina

5 Magister en Matemática y Estadística, Departamento de Fisicomatemática, Facultad de Farmacia y Bioquimica, Universidad de Buenos Aires, Ciudad Autónoma de Buenos Aires, Argentina 
6 Médica Pediatra, Hospital Notti, Mendoza, Argentina

7 Médica Clínica, especialista en Diabetes, Instituto Médico Catamarca, Rosario, Santa Fe, Argentina

8 Profesora Titular de Nutrición y Educación para la Salud, Facultad de Medicina, Universidad Nacional del Nordeste (UNNE), Corrientes, Argentina

9 Médica Clínica, especialista en Nutrición y Diabetes, Servicio de Medicina Interna y Nutrición, Hospital General de Agudos Carlos G. Durand, Ciudad Autónoma de Buenos Aires, Argentina

10 Bioquímica, Directora General Laboratorio Hidalgo, Provincia de Buenos Aires, Martínez, Argentina

11 Director Bioquímico, Laboratorio Hidalgo, Provincia de Buenos Aires, Martínez Argentina

12 Estudiante de Medicina, Universidad Nacional de Rosario, Rosario, Santa Fe, Argentina

13 Estudiante de Medicina, Instituto Universitario Italiano de Rosario, Santa Fe, Rosario, Argentina

14 Médica Pediatra, Jefa del Servicio de Pediatría, Hospital Regional Ushuaia, Ushuaia, Tierra del Fuego, Argentina

15 Médica Pediatra, Servicio de Pediatría, Hospital Regional Ushuaia, Ushuaia, Tierra del Fuego, Argentina

16 Médica Pediatra, Servicio de Pediatría, Hospital Regional Ushuaia, Responsable del Programa de Salud Escolar, Ushuaia, Tierra del Fuego, Argentina

17 Bioquímica, especialista en Endocrinología, Laboratorio del Hospital Regional Ushuaia, Ushuaia, Tierra del Fuego, Argentina
18 Bioquímica, Jefa del Servicio de Química Clínica, Laboratorio del Hospital Regional Ushuaia, Ushuaia, Tierra del Fuego, Argentina

19 Técnica, Laboratorio del Hospital Regional Ushuaia, Ushuaia, Tierra del Fuego, Argentina

20 Médica de Planta, Endocrinología Infantil, Servicio de Endocrinología, Hospital Notti, Mendoza, Argentina

21 Médica Residente, Servicio de Endocrinología, Hospital Notti, Mendoza, Argentina

22 Médica Residente, Medicina Familiar, Área de Salud, Maipú, Mendoza, Argentina

23 Bioquímico, Jefe de Residentes, Laboratorio Hospital Notti, Mendoza, Argentina

24 Bioquímica Residente, Laboratorio Hospital Notti, Mendoza, Argentina

\author{
Contacto de la autora: Valeria Hirschler \\ E-mail: vhirschler@gmail.com \\ Correspondencia: Maipú 812 50 "M" (C1006ACL), Ciudad \\ Autónoma de Buenos Aires, Argentina \\ Fecha de trabajo recibido: 14/06/20 \\ Fecha de trabajo aceptado: 22/07/20
}

Conflictos de interés: los autores declaran que no existe conflicto de interés

\section{INTRODUCCIÓN}

El aumento en la prevalencia de sobrepeso (SP) y obesidad (OB) en niños se describió tanto en países desarrollados como en desarrollo. Desde la década de 1980 la prevalencia de OB ha aumentado del 6,5 al 18,0\% en niños escolares de 6 a 11 años en los EE.UU. . Actualmente un tercio de los niños presenta SP/OB ${ }^{2}$. La prevalencia de SP y OB en la población de 5 a 17 años en Argentina fue del 41,1y 20,4\% sin diferencias significativas por región, nivel educativo, cobertura de salud ni ingresos, según la Segunda Encuesta Nacional de Nutrición y Salud del Ministerio de Salud de la Nación.

Observamos una prevalencia similar de SP/OB en niños de escuelas primarias de Buenos Aires ${ }^{3}$. El incremento de peso se debe a un balance energético positivo ya sea por un aumento en la ingesta calórica y/o una disminución del gasto calórico. El estilo de vida poco saludable -consumo de bebidas azucaradas, falta de ingesta de desayuno, bajo consumo de leche, baja ingesta de verduras y frutas, mayor tiempo frente a la televisión, así como la presencia del televisor en la habitación del niño- se ha relacionado con la mayor prevalencia de SP/OB ${ }^{4}$.

El "síndrome del bebé pequeño" (peso al nacer, PN) se asoció al desarrollo posterior de SP/OB, así como a enfermedades cardiometabólicas ${ }^{5}$. Además, se ha demostrado que el PN bajo y alto y posterior SP/OB y riesgo cardiometabólico tenían una distribución en $U$ o J invertida ${ }^{6}$. Sin embargo, la mayoría de los estudios publicados se ha centrado principalmente en el bajo PN sin considerar a las personas con alto $P N^{78}$. Los niños con alto $P N$, y que además están expuestos a un ambiente intrauterino de obesidad materna, tienen aún mayor riesgo cardiometabólico?.

Un estudio previo realizado en los suburbios de Buenos Aires en más de 1.000 escolares de 9 años, demostró que el alto PN se asoció con SP/OB y síndrome metabólico ${ }^{10}$. Sin embargo, el bajo PN fue un factor protector de $\mathrm{SP} / \mathrm{OB}^{10}$. Hasta donde se conoce no, existen estudios previos sobre el PN y el índice de masa corporal (IMC) en escolares de 9 años que viven en diferentes áreas de Argentina.

\section{OBJETIVOS}

El objetivo de este estudio fue determinar la asociación entre PN y OB en escolares de 9 años de tres regiones de Argentina.

\section{MATERIALES Y MÉTODOS}

Este estudio transversal se realizó en tres áreas distribuidas dentro de Argentina y lo aprobó el Comité de Ética de la Universidad de Buenos Aires. Cada tutor y niño dieron su consentimiento informado por escrito después de una explicación y antes de su inicio. Este trabajo es parte de un estudio multicéntrico (DIOSES) realizado en tres áreas de 
Argentina distribuidas de la siguiente manera: Rosario (centro), Mendoza (oeste) y Ushuaia (sur).

Para la selección de la muestra se aplicó un muestreo por conglomerados en dos etapas. El marco fueron las escuelas enumeradas en cada área y se tomó el número de estudiantes para cada una. En la primera etapa, las escuelas se seleccionaron con el método de muestreo proporcional al tamaño de Sampford, que se programó en R. El número de escuelas seleccionadas se determinó previamente para que el número de estudiantes incluidos garantice un error de aproximadamente un 5\% en cada área, con un $95 \%$ de confianza, considerando el factor de corrección para la población finita.

Dentro de las escuelas, los estudiantes se seleccionaron al azar con un generador de números aleatorios disponibles en el Epidat 3.1. Dado que en un estudio previo en los escolares de los suburbios de Buenos Aires la prevalencia de SP/OB fue del $33 \% \%^{3}$, se estimó un tamaño muestral para lograr un error inferior a 0,05. Examinamos 1.131 niños, con lo cual el error global de la muestra fue inferior a 0,05.

Las características socioeconómicas incluyeron el nivel de educación y la presencia o ausencia de heladera o el piso de tierra en la casa. El Instituto Nacional de Estadística y Censos de Argentina (INDEC) utiliza estos dos indicadores para identificar a las familias de bajo nivel socioeconómico ${ }^{11}$. Los médicos completaron un cuestionario sobre hábitos de vida previamente validado ${ }^{12}$ que incluía: consumo diario de verduras o frutas frescas, vasos de leche, bebidas azucaradas, televisión y la presencia de televisor en la habitación del niño ${ }^{12}$.

La talla y el peso se midieron con ropa ligera y sin zapatos. El IMC se calculó como el peso (en kilogramos, $\mathrm{kg}$ ) dividido por la altura (en metros, $\mathrm{m}$ ) al cuadrado. También se determinaron las puntuaciones z de IMC (IMC-z $)^{13}$. Los niños se clasificaron como de bajo peso $(<5$ percentil), peso normal $(5$ a $<85$ percentil), SP (85 a $<95$ percentil) u OB ( $\geq 95$ percentil), según las normas de los Centros para el Control y la Prevención de Enfermedades (CDC) de EE.UU. ${ }^{13}$. Los profesionales médicos certificados midieron la presión arterial durante el examen físico ${ }^{14}$.

La tasa de respuesta individual global de todo el grupo fue del 94\%. Los criterios de exclusión fueron: 1) falta de datos de medidas antropométricas o presión arterial; 2) presencia de una enfermedad crónica o uso de medicamentos que afectarían la presión arterial; 3) embarazo autoinformado en el momento del examen. Los participantes incluidos en la muestra no tuvieron diferencias significativas en el nivel socioeconómico, edad, IMC y circunferencia de cintura (CC) con quienes fueron excluidos debido a la falta de datos.

\section{Análisis estadísticos}

Las estadísticas descriptivas para variables se presentan como valores medios $\pm \mathrm{DE}$. La prueba de chi cuadrado se utilizó para comparar proporciones. Cuando más del $20 \%$ de las células esperaban frecuencias $<5$, se utilizó la prueba exacta de Fisher. La prevalencia observada se presentó en porcentajes. Al comparar dos grupos con datos distribuidos normalmente se realizó la prueba t de Student. Las variables con una distribución sesgada se transformaron logarítmicamente para el análisis. El ajuste de Bonferroni se efectuó cuando se verificaron muchas comparaciones. Para la prueba ANOVA se probó la homogeneidad de las variaciones. Cuando no fue validada, se realizó la prueba Brown Forsythe.

El objetivo principal del estudio fue determinar la asociación entre PN y la adiposidad ajustadas por variables confundentes. Se realizaron análisis de regresión logística múltiple para determinar la asociación entre PN y OB edad, sexo y estilo de vida. Los valores de $p<0,05$ se consideraron estadísticamente significativos. Los análisis se analizaron con el paquete de software estadístico IBM SPSS versión 22.0 (IBM Corp., Armonk, NY, EE.UU.).

\section{RESULTADOS}

Se examinaron niños $(n=1.131,505$ masculinos) de 8,8 $\pm 2,1$ años de edad promedio de ocho escuelas primarias en cuatro áreas de Argentina, entre abril y septiembre de 2019 (Tabla 1). Nivel socioeconómico: ninguna de las familias tenía piso de tierra y sólo el 2,7\% carecía de heladera. Las madres tenían un nivel de educación formal significativamente mayor que el de los padres: el $75,7 \%$ de las madres vs el $68,7 \%$ de los padres tenía educación secundaria o más.

Las características clínicas de la muestra según sexo se describen en la Tabla 1. Los niños se dividieron según el género. No hubo diferencia significativa en la edad, peso, talla, IMC, z-IMC y presión arterial (PA) sistólica y diastólica entre ambos géneros. Sin embargo, los varones tenían una CC (diferencia de medias 1,86; 95\% IC 0,55-3,16; p0,005) y un PN (diferencia de medias 1,17; 95\% IC 0,09-0,24; $p<0,001$ ) significativamente mayor que las niñas. 


\begin{tabular}{|l|c|c|c|}
\hline & Varones $(\mathbf{N}=\mathbf{5 0 5})$ & Mujeres $(\mathbf{N}=\mathbf{6 2 6}))$ & Total (N=1.131) \\
\hline Edad (años) & $8,87 \pm 2,03$ & $8.74 \pm 2,18$ & $8.8 \pm 2,11$ \\
\hline PN $(\mathrm{kg})$ & $3,39 \pm 0,55$ & $3,22 \pm 0,58^{*}$ & $3,29 \pm 0,57$ \\
\hline Talla $(\mathrm{m})$ & $1,36 \pm 0,13$ & $1,35 \pm 0,14$ & $1,35 \pm 0,14$ \\
\hline Peso $(\mathrm{kg})$ & $36,34 \pm 12,97$ & $35,22 \pm 12,89$ & $35,72 \pm 12,93$ \\
\hline IMC & $19,23 \pm 4,01$ & $18,85 \pm 3,87$ & $19,02 \pm 3,94$ \\
\hline z-IMC & $0,81 \pm 1,17$ & $0,67 \pm 1,01$ & $0,73 \pm 1,08$ \\
\hline CC $(\mathrm{cm})$ & $67,06 \pm 11,35$ & $65,2 \pm 10,78^{*}$ & $66,03 \pm 11,07$ \\
\hline Frecuencia cardíaca & $90,62 \pm 16,23$ & $91,58 \pm 16,25$ & $91,16 \pm 16,23$ \\
\hline PA sistólica $(\mathrm{mmHg})$ & $102,05 \pm 13,64$ & $101,23 \pm 13,29$ & $101,59 \pm 13,45$ \\
\hline PA diastólica $(\mathrm{mmHg})$ & $62,98 \pm 10,78$ & $63,2 \pm 10,44$ & $63,1 \pm 10,59$ \\
\hline
\end{tabular}

Los datos se presentan como media $\pm D E$. La puntuación Z es una medida cuantitativa de la desviación de una variable específica tomada de la media de esa población. Los CDC z-IMC tienen en cuenta la edad y el sexo.

* Significancia: $p<0,01$.

PN: peso al nacer; IMC: índice de masa corporal; z-IMC: IMC puntaje z; CC: circunferencia cintura; PA: presión arterial.

Tabla 1: Características de la muestra según sexo.

\section{Características clínicas según peso de nacimiento}

La prevalencia de PN bajo $(<2.500 \mathrm{~g})$ y alto $(>4.000 \mathrm{~g})$ fue de $6,2 \%(n=70)$ y $7,3 \%(n=82)$ respectivamente. El 44\% (31) de los niños de 9 años con bajo PN bebieron tres o más vasos de leche por día vs el $28 \%(23)$ de alto PN ( $p<0,01)$. El $32 \%$ (12) de los niños de 9 años con PN bajo tenía el televisor en el cuarto vs el $48 \%$ (14) de PN alto $(p<0,01)$. El 55\% (45) de los niños con alto PN presentaba SP/OB a los 9 años vs el $33 \%$ de los niños con bajo PN (23) $(p<0,01)$.

Las características clínicas de la muestra según PN se describen en la Tabla 2. No hubo diferencia significativa en la edad, peso talla y PA sistólica y diastólica según PN. Sin embargo, el peso, IMC, z-IMC y CC de los niños de 9 años aumentaba significativamente conforme se incrementaba el PN. El 33\% (23) de los niños de 9 años con PN bajo vs $55 \%(45)$ de PN alto presentaba SP/OB $(p<0,01)$.

\begin{tabular}{|l|c|c|c|}
\hline & Bajo PN (0,85-2,50 kg) & Normo PN (2,53-3,98 kg) & Alto PN (4-4,70 kg) \\
\hline Edad (años) & $8,86 \pm 2,08$ & $8,7 \pm 2,15$ & $8,99 \pm 2,15$ \\
\hline Talla $(\mathrm{m})$ & $1,34 \pm 0,13$ & $1,34 \pm 0,14$ & $1,39 \pm 0,13$ \\
\hline Peso $(\mathrm{kg})^{*}$ & $32,71 \pm 11,84$ & $35,27 \pm 13$ & $40,1 \pm 13,72$ \\
\hline IMC* $^{*}$ & $17,87 \pm 3,33$ & $18,93 \pm 3,94$ & $20,28 \pm 4,15$ \\
\hline z-IMC* & $0,33 \pm 1,13$ & $0,73 \pm 1,05$ & $1,07 \pm 1,12$ \\
\hline CC* & $63,54 \pm 9,55$ & $65,5 \pm 11,02$ & $70,19 \pm 11,48$ \\
\hline Frecuencia cardíaca & $86,12 \pm 14,81$ & $91,66 \pm 16,18$ & $10,1 \pm 11,11$ \\
\hline PA sistólica $(\mathrm{mmHg})$ & $102,91 \pm 16,78$ & $101,85 \pm 13,24$ & $63,14 \pm 9,65$ \\
\hline PA diastólica $(\mathrm{mmHg})$ & $63,6 \pm 13,04$ & $63,28 \pm 10,31$ & $63,1 \pm 10,59$ \\
\hline PA diastólica $(\mathrm{mmHg})$ & $62,98 \pm 10,78$ & $63,2 \pm 10,44$ & \\
\hline
\end{tabular}

Los datos se presentan como media $\pm D E$. La puntuación Z es una medida cuantitativa de la desviación de una variable específica tomada de la media de esa población. Los CDC z-IMC tienen en cuenta la edad y el sexo.

* Significancia: $p<0,01$.

IMC: índice de masa corporal; z-IMC: IMC puntaje z; CC: circunferencia cintura; PA: presión arterial.

Tabla 2: Características de la muestra según PN.

\section{SP/OB vs normopeso en los niños}

El 42,9\% (485) de los niños presentaba SP/OB. La prevalencia de SP/OB fue significativamente mayor en los niños ( $n=236,46,7 \%$ ) que en las niñas $(n=249,39,8 \%)$.
Las características clínicas de la muestra según sexo se describen en la Tabla 3. Los niños con SP/OB tenían mayor peso al nacer y eran más altos; además presentaron valores significativamente más altos de CC y PA sistólica y diastólica. 
Las diferencias significativas de las diferencias de las medias y $95 \%$ de IC en niños con normopeso vs niños con sobrepeso/obesidad fueron las siguientes: PN (diferencia de medias -0,14; $95 \%$ IC $-0,22$ a $-0,07 ; p<0,001$ ), IMC (diferencia de medias $-5,71 ; 95 \%$ IC $-6,06$ a-5,35; $p<0,01)$, peso (diferencia de medias -12,85; 95\% IC -0,05 a -0,02; $p<0,001$ ), talla (diferencia de medias $-0,03 ; 95 \%$ IC $-6,06$ a $-5,35 ; p<0,01)$, CC (diferencia de medias $-13,08 ; 95 \%$ IC $-14,15$ a-11,93; $p<0,001$ ), PAS (diferencia de medias $-3,12$; 95\% IC $-4,71$ a-1,54; $\mathrm{p}<0,001$ ) y $\mathrm{PAD}$ (diferencia de medias $-1,78$; 95\% IC $-3,04 a-5,28 ; p<0,001)$.

Los niños con SP/OB consumían menos tazas de leche por día y omitían desayuno con más frecuencia comparados con aquellos con peso normal. Sin embargo, no hubo diferencias significativas entre los niños con SP/OB y de peso normal en las horas que miraban televisión, en las porciones de ingesta de verduras y frutas, o en la presencia de TV en la habitación.

\begin{tabular}{|l|r|r|}
\hline & Normopeso & \multicolumn{1}{c|}{ SP/OB } \\
\hline Edad (años) & $8,8 \pm 2,07$ & $8,79 \pm 2,17$ \\
\hline Vegetales/frutas (<3/día) & $36,60 \%$ & $40,10 \%$ \\
\hline Leche (>3/día)** & $27,40 \%$ & $16,10 \%$ \\
\hline Falta de desayuno** & $13,80 \%$ & $24,30 \%$ \\
\hline PN* & $3,23 \pm 0,58$ & $3,38 \pm 0,55$ \\
\hline Talla (m)** & $1,34 \pm 0,13$ & $1,37 \pm 0,14$ \\
\hline Peso $(\mathrm{kg})^{* *}$ & $30,2 \pm 8,34$ & $43,06 \pm 14,25$ \\
\hline IMC & $16,56 \pm 1,7$ & $22,27 \pm 3,69$ \\
\hline z-IMC** & $-0,01 \pm 0,79$ & $1,72 \pm 0,46$ \\
\hline CC (cm)** & $60,47 \pm 6,8$ & $73,55 \pm 11,29$ \\
\hline Frecuencia cardíaca & $90,96 \pm 16,61$ & $91,46 \pm 15,69$ \\
\hline PA diastólica $(\mathrm{mmHg})^{* *}$ & $100,26 \pm 13,43$ & $103,38 \pm 13,27$ \\
\hline PA sistólica $(\mathrm{mmHg})^{* *}$ & $62,34 \pm 10,82$ & $64,12 \pm 10,2$ \\
\hline
\end{tabular}

Los datos se presentan como media $\pm D E$ y porcentaje (\%). La puntuación Z es una medida cuantitativa de la desviación de una variable específica tomada de la media de esa población. Los CDC z-IMC tienen en cuenta la edad y el sexo.

Significancia: ${ }^{*}<0,05 ;{ }^{*} p<0,01$.

PN: peso al nacer; IMC: índice de masa corporal; z-IMC: IMC puntaje z; CC: circunferencia de cintura; PA: presión arterial.

Tabla 3: Características clínicas según SP/OB.

\section{Asociaciones multivariadas entre PN y adiposidad}

Regresión múltiple

- Lineal: el análisis de regresión lineal múltiple mostró una relación lineal significativa y positiva entre el IMC en niños de 9 años y el PN (Beta=0, 19; $R 2=0.10$ ) ajustado por edad, sexo, ingesta de le- che y de frutas. Cuando el IMC se reemplazó por la circunferencia de la cintura, los resultados fueron también significativos (Beta=0,18; R2=0.10).

- Logística: en modelos de regresión logística múltiple, el PN bajo demostró ser un factor protector para la OB (OR, 0.41 [IC del 95\%: 0,19-0,92]), mientras que el PN alto se asoció directamente con la OB ajustado por edad y sexo (OR, 2.48 [95\% IC 1,53-4,02]) (Tabla 4).

\begin{tabular}{|l|c|c|c|c|}
\hline & Significancia & OR & \multicolumn{2}{|c|}{$\mathbf{9 5} \% \mathbf{I C}$ para $\operatorname{Exp}$ (B) } \\
\hline Edad (años) & 0,54 & 1,04 & 0,92 & 1,16 \\
\hline Sexo & 0,01 & 2,11 & 1,2 & 3,65 \\
\hline Obesidad & 0,03 & 0,41 & 0,19 & 0,92 \\
\hline
\end{tabular}

$\checkmark$ dependiente bajo PN. Curva ROC: curva área bajo la curva $0,62795 \%$ IC $(0,594-0,658)$.

\begin{tabular}{|l|c|c|c|c|}
\hline & Significancia & OR & \multicolumn{2}{|c|}{$\mathbf{9 5} \%$ IC para Exp (B) } \\
\hline Edad (años) & 0,33 & 1,06 & 0,95 & 1,16 \\
\hline Sexo & 0,01 & 0,52 & 1,2 & 0,83 \\
\hline Obesidad & 0,00 & 2,48 & 1,53 & 0,92 \\
\hline
\end{tabular}

$\checkmark$ dependiente Bajo PN. Curva ROC: curva área bajo la curva 0,627 95\% IC (0,594-0,658).

Tabla 4: Regresión logística múltiple.

\section{DISCUSIÓN}

Este estudio multicéntrico realizado en escolares de tres áreas de Argentina demostró que la $\mathrm{OB}$ se asoció con alto peso al nacer. Los niños OB tenían hábitos de vida menos saludables ya que salteaban sus desayunos y la ingesta de leche diaria era menor respecto de los niños normopeso. En el análisis multivariado, la adiposidad se asoció de manera significativa y directa con el PN, lo que sugiere que la adiposidad podría ser el factor principal asociado a un mayor PN. Por el contrario, el bajo PN se asoció en forma inversa con la adiposidad lo que indicaría que el bajo PN podría ser un factor protector de la adiposidad. Un estudio previo realizado por nuestro grupo demostró que el alto PN se asoció a la adiposidad y el síndrome metabólico en niños ${ }^{10}$. Sin embargo, el estudio anterior incluía sólo escolares de los suburbios de Buenos Aires, mientras que el presente incluye a niños de diferentes áreas de Argentina (centro, sur y oeste), lo que aumenta la influencia del PN y futura adiposidad.

La "hipótesis de los orígenes fetales" respalda la relación entre el crecimiento fetal reducido y la posterior OB, así como el mayor riesgo cardiometabólico ${ }^{5,6}$. Se describieron resultados mixtos so- 
bre la asociación entre bajo PN y futuras enfermedades cardiovasculares y diabetes mellitus tipo 2 (DM2) en la edad adulta7,15,16. Se ha criticado a David Barker, padre de esta hipótesis ${ }^{17}$, de llegar a estas conclusiones con convicción entusiasta, pero con sesgos importantes en la selección de las variables confundentes ${ }^{7}$. En efecto, además de la potencial influencia del error aleatorio en las conclusiones del estudio de Baker, los métodos empleados para la selección de las variables confusoras han sido objeto de debate. La posibilidad de dejar fuera del ajuste multivariado a algunas covariables potencialmente importantes, podría haber afectado la validez de las conclusiones del estudio?.

Un trabajo brasileño demostró que adolescentes con alto PN tenían un riesgo de dos a tres veces mayor de presentar OB y otros marcadores cardiometabólicos que aquellos con PN normal, lo cual sugiere que la excesiva adiposidad durante la vida fetal podría conducir a alteraciones cardiometabólicas durante la adolescencia ${ }^{18}$. Además, un metaanálisis demostró que el alto PN se asociaba al doble de riesgo de presentar SP en el futuro, independientemente de la etnia, el género, la clase socioeconómica y el peso materno ${ }^{19}$. Adolescentes en Jerusalén, con PN alto, tenían un riesgo dos veces y medio mayor de desarrollar hipertensión comparados con adolescentes con PN normal ${ }^{20}$.

En los suburbios de Buenos Aires, nuestro grupo detectó una asociación significativa entre alto PN y síndrome metabólico en la infancia ${ }^{10}$. En Lituania se estudió en 4.600 adolescentes que el alto PN se asociaba al aumento de PA, particularmente en aquellos que tenían SP/OB en la adolescencia ${ }^{21}$. De acuerdo con estos resultados, este estudio demostró que el alto PN se asociaba a adiposidad. En un estudio efectuado en 12 países en niños de 9-11 años se determinó que la OB infantil se asoció significativamente con el alto PN (OR 2.08; IC del 95\%: 1,47-2,93) respecto del grupo de PN adecuado22. Más aún, el OR del trabajo realizado en 12 países es muy similar al de nuestro trabajo (OR 2.48) elaborado en diferentes regiones de nuestro país. La diversidad genética y epigenética probablemente podría asociarse al desarrollo de SP/OB durante la infancia en niños con PN alto ${ }^{23}$. Sin embargo, el análisis de todo el genoma aún no se realizó23. Esto sugiere que hay que seguir de cerca a los niños con alto PN y aconsejar cambios en el estilo de vida para prevenir futuras enfermedades cardiometabólicas.
Hay que reconocer varias limitaciones. Este estudio es transversal, no puede determinarse una relación causa-efecto. Los niños con alto PN son un grupo heterogéneo que comprende salud materna/embarazo, diabetes gestacional materna u obesidad materna, o niños grandes normales con PN alto acorde a sus antecedentes genéticos. Estos datos no se incorporaron al estudio debido a la falta de información.

A pesar de las limitaciones mencionadas anteriormente, las fortalezas de nuestro estudio incluyen una gran cantidad de escolares aparentemente normal en diferentes áreas de Argentina con una alta tasa de respuesta. Además, utilizamos modelos de regresión múltiple para hacer los ajustes necesarios para las variables de confusión.

\section{CONCLUSIONES}

Nuestros datos indican que un alto $\mathrm{PN}$, pero no el bajo PN, se asocia con OB en niños en edad escolar de 9 años. Además, el bajo PN está inversamente asociado con OB. Deberían incorporarse hábitos de vida saludable en las familias a fin de prevenir y tratar la SP/OB en los niños, especialmente en aquellos con alto PN. De esta manera, deberían realizarse futuros estudios longitudinales para determinar el impacto del alto PN en la salud poblacional.

\section{Agradecimientos:}

El Proyecto DIOSES ha sido parcialmente financiado por la Sociedad Argentina de Diabetes. El Laboratorio Hidalgo procesó las muestras de laboratorio en forma gratuita. Agradecemos especialmente a todos los niños participantes y sus familias.

\section{BIBLIOGRAFÍA}

1. Ogden CL, Carroll MD, Kit BK, Flegal KM. Prevalence of obesity and trends in body mass index among US children and adolescents, 1999-2010. JAMA 2012; 307:483-490.

2. Kumar S, Kelly AS. Review of childhood obesity: from epidemiology, etiology and comorbidities to clinical assessment and treatment. Mayo Clin Proc 2017; 92:251-265.

3. Hirschler V, Oestreicher K, Maccallini G, Aranda C. Relationship between obesity and metabolic syndrome among Argentinean elementary schoolchildren. Clin Biochem 2010; 43:435-41.

4. Taber DR, Chriqui JF, Powell L, Chaloupka FJ. Association between state laws governing school meal nutrition content and student weight status: implications for new USDA school meal standards. JAMA Pediatr 2013; 167: 513-519.

5. Barker DJ, Hales CN, Fall CHD, Osmond C, Phipps K, et al.Type 2 (non-insulin-dependent) diabetes mellitus, hypertension and hyperlipidaemia (syndrome $\mathrm{X}$ ): relation to reduced fetal growth. Diabetologia 1993; 36: 62-67.

6. Gluckman PD, Hanson MA, Cooper C, Thornburg KL. Effect of in utero and early-life conditionson adult health and disease. $\mathrm{N}$ Engl J Med 2008; 359:61-73. 
7. Huxley R, Owen CG, Whincup PH, Cook DG, Rich-Edwards J, Smith GD, Collins R. Isbirth weight a risk factor for ischemic heart disease in later life? Am J Clin Nutr 2002; 85:807-814.

8. Huxley RR, Shiell AW, Law CM. The role of size at birth and postnatal catch-up growth in determining systolic blood pressure: a systematic review of the literature. J Hypertension 2000; 18:815-831.

9. Boney CM, Verma A, Tucker R, Vohr BR. Metabolic syndrome in childhood: association with birth weight, maternal obesity, and gestational diabetes mellitus. Pediatrics 2005; 115:e290-6. DOI: 10.1542/peds.2004-1808.

10. Hirschler V, Bugna J, Roque M, GilliganT, González C. Does low birth weight predict obesity/overweight and metabolic syndrome in elementary school children? Arch Med Res 2008; 39:796802. DOI: 10.1016/j.arcmed.2008.08.003.

11. Instituto Nacional de Estadística y Censos de la República Argentina, 2010. Disponible en: http://www.censo2010.indec.gov. ar/. Acceso: 3/09/15. Último acceso: 03/19.

12. Hirschler V, Buzzano K, Erviti A, Ismael N, Silva S, Dalamon R. Overweight and lifestyle behaviors of low socioeconomic elementary school children in Buenos Aires. BMC Pediatr 2009; 24:9-17.

13. Kuczmarski RJ, Ogden CL, Guo SS, et al. 2000 CDC growth charts for the United States: methods and development. Vital Health Stat 11, 2002; 246:1-190. Disponible en: http://www.cdc. gov/GROWTHCHARTS.

14. Flynn JT, Kaelber DC, Baker-Smith CM, et al; Subcommittee on screening and management of high blood pressure in children. Clinical practice guideline for screening and management of high blood pressure in children and adolescents. Pediatrics 2017; 140:e20171904.

15. Huxley R, Owen CG, Whincup PH, Cook DG, Colman S, et al.
Birth weight and subsequent cholesterol levels. Exploration of the "fetal origins" hypothesis. JAMA 2004; 292:2755-2764.

16. Harder T, Rodekamp E, Schellong K, Dudenhausen JW, Plagemann A. Birth weight and subsequent risk of type 2 diabetes: a meta-analysis. Am J Epidemiol 2007; 165:849-857.

17. Barker DJP, Bull AR, Osmond C, Simmonds SJ. Fetal and placental size and risk of hypertension in adult life. BMJ 1990; 301:25962.

18. Sousa MA, Guimarães IC, Daltro C, Guimarães AC. Association between birth weight and cardiovascular risk factors in adolescents. Arq Bras Cardiol 2013; 101:9-17.

19. Schellong K, Schulz S, Harder T, Plagemann A. Birth weight and long-term overweight risk: systematic review and a metaanalysis including 643.902 persons from 66 studies and 26 countries globally. PLoS One 2012; 7:e47776. Doi: 10.1371/ journal. pone.0047776.

20. Seidman DS, Laor A, Gale R, Stevenson DK, Mashiach S, Danon YL. Birth weight, current body weight, and blood pressure in late adolescence. BMJ 1991; 302:1235-1237.

21. Kuciene R, Dulskiene V, Medzioniene J. Associations between high birth weight, being large for gestational age, and high blood pressure among adolescents: a cross-sectional study Eur J Nutr 2018; 57:373-381. DOI: 10.1007/s00394-016-1372-0.

22. Qiao Y, Ma j, Wang Y, Li W, Katzmarzyk PT, Chaput JP, Hu G. ISCOLE Research Group Birth weight and childhood obesity: a 12-country study. Int J Obes Suppl 2015; 5:S74-9. DOI: 10.1038/ ijosup.2015.23.

23. Diels S, Vanden Berghe W, Van HulW. Insights into the multifactorial causation of obesity by integrated genetic and epigenetic analysis. Obesity Reviews 2020;1-16. 